\title{
Detailed Evolution of Degree Distributions in Residual Graphs with Joint Degree Distributions
}

\author{
Takayuki NOZAKI ${ }^{\dagger \mathrm{a})}$, Nonmember, Kenta KASAI ${ }^{\dagger \mathrm{b})}$, Tomoharu SHIBUYA ${ }^{\dagger \dagger \mathrm{c})}$, Members, $^{\dagger}$ \\ and Kohichi SAKANIWA ${ }^{\dagger \mathrm{d})}$, Fellow
}

\begin{abstract}
SUMMARY Luby et al. derived evolution of degree distributions in residual graphs for irregular LDPC code ensembles. Evolution of degree distributions in residual graphs is important characteristic which is used for finite-length analysis of the expected block and bit error probability over the binary erasure channel. In this paper, we derive detailed evolution of degree distributions in residual graphs for irregular LDPC code ensembles with joint degree distributions.

key words: detailedly represented irregular LDPC code ensembles, evolution of degree distributions in residual graphs, peeling algorithm, binary erasure channel
\end{abstract}

\section{Introduction}

Gallager invented low-density parity-check (LDPC) codes [1] in 1963. LDPC codes are linear codes defined by sparse bipartite graphs. Luby et al. introduced irregular LDPC code ensembles [2] defined by some distributions which represent the degrees of nodes. We can obtain flexibility in balancing requirement that variable nodes want high degrees and check nodes want low degrees.

Luby et al. introduced the peeling algorithm (PA) [4], [7] for the binary erasure channel (BEC). PA is an iterative algorithm which is defined on Tanner graphs. As PA proceeds edges and nodes are removed, and the decoding is successful if the graph vanished. The residual graphs consist nodes and edges that are still unknown at each iteration. Evolution of degree distributions in residual graphs can be used for finite-length scaling of decoding performance of belief propagation (BP). Luby et al. [4] derived it for irregular LDPC code ensembles. Amraoui [5] showed that distributions of the number of check nodes of degree one in the residual graph convergences weakly to a Gaussian as blocklength tends to infinity and that block and bit error probability of finite-length LDPC codes are derived by the average and the variance of the number of check node of degree one in the residual graph.

The check distributions of the optimized finite-length

Manuscript received February 1, 2008.

Manuscript revised April 18, 2008.

${ }^{\dagger}$ The authors are with the Dept. of Communications and Integrated Systems, Tokyo Institute of Technology, Tokyo, 152-8552 Japan.

${ }^{\dagger \dagger}$ The author is with the Dept. of Mathematics, Sophia University, Tokyo, 102-8554 Japan.

a)E-mail: nozaki@comm.ss.titech.ac.jp

b)E-mail: kenta@comm.ss.titech.ac.jp

c) E-mail: tshibuya@sophia.ac.jp

d)E-mail: sakaniwa@ comm.ss.titech.ac.jp

DOI: 10.1093/ietfec/e91-a.10.2737
LDPC codes in [5] tend to have different degrees like $0.390753 x^{4}+0.361589 x^{5}+0.247658 x^{9}$ while optimized infinite-length LDPC codes in [6] have concentrated degree distributions of check nodes like $x^{9}$. It is known that irregular LDPC codes defined by joint degree distributions introduced in [3] performs well with such non-concentrated check degree distributions.

In this paper, as the first step to optimize finite-length irregular LDPC codes with joint degree distributions, we investigate evolution of residual graphs in PA. Furthermore, we give a graphical determination of successful decoding.

\section{Preliminaries}

\subsection{Channel Model}

In this paper, we consider the binary erasure channel (BEC). A channel input, denoted by $X$, is binary $X \in\{0,1\}$. The corresponding output $Y$ takes on values in the alphabet $\{0,1, ?\}$, where ? indicating an erasure. Let $\epsilon$ be the erasure probability, i.e. $\epsilon=\operatorname{Pr}(Y=$ ? $\mid X=x)$ and $1-\epsilon=\operatorname{Pr}(Y=x \mid X=x)$.

\subsection{Peeling Decoder}

The peeling algorithm (PA) is an iterative decoding algorithm. It works on Tanner graphs of LDPC codes over BEC. $\mathrm{BP}$ and PA are completely equivalent in terms of their performance for BEC. Each check node has a one-bit memory cell. Residual graphs are defined as the graphs which consist nodes and edges that are still unknown at each iteration.

(1) Initialization

Variable nodes receive channel outputs: known messages ' 0 ' and ' 1 ' or an erasure '?.' The variable nodes with known received messages, send their value to check nodes by edges. They and all edges connecting to them are removed from the graph. Check nodes store the sum of the receiving values in their memory cells.

\section{(2) Iteration}

The decoder uniformly chooses a check node of degree one in the residual graph. The check node sends the value in its memory cell to the variable node connected to it. The variable node propagates this value to all check nodes connected to it and is removed with its edges. Each check node overwrites its memory cell with the sum of the receiving value 
and the value of its memory cell.

\section{(3) Decisions}

If the decoder does not find any check node of degree one in the residual graph, then the decoding halts. If the residual graph is empty, decoding is succeeds, otherwise it fails and the variable nodes in the residual graph form a stopping set.

2.3 Detailedly Represented Irregular LDPC Code Ensembles (Detailedly Represented Ensembles)

In this paper, we use detailedly represented ensembles. Let $b_{i}$ and $d_{j}$ be a set of variable nodes of degree $i$ and a set of check nodes of degree $j$, respectively. Let $1_{\mathrm{m}}$ and $\mathrm{r}_{\mathrm{m}}$ be maximum degree of variable nodes and check nodes, respectively. Let B and D be

$$
\mathrm{B}:=\left\{b_{2}, b_{3}, \cdots, b_{\mathrm{l}_{\mathrm{m}}}\right\}, \quad \mathrm{D}:=\left\{d_{2}, d_{3}, \cdots, d_{\mathrm{r}_{\mathrm{m}}}\right\} .
$$

A function $\pi: \mathrm{B} \times \mathrm{D} \rightarrow[0,1]$ is said to be a joint degree distribution, if $\sum_{b_{i} \in \mathrm{B}} \sum_{d_{j} \in \mathrm{D}} \pi(i, j)=1$.

A detailedly represented ensemble $C(n, \pi)$ [3] is defined as an ensemble which consists of all bipartite graphs $\mathrm{G}=(\mathrm{V} \cup \mathrm{C}, \mathrm{E})$ such that $n=|\mathrm{V}|$ and $\pi(i, j)=\left|\mathrm{E}_{i, j}\right| /|\mathrm{E}|$ for all pair of $b_{i} \in \mathrm{B}, d_{j} \in \mathrm{D}$, where $\mathrm{E}_{i, j}$ represents a set of edges connected to variable nodes of degree $i$ and check nodes of degree $j$. Moreover we define $\lambda_{i}:=\sum_{j} \pi(i, j), \rho_{j}:=$ $\sum_{i} \pi(i, j), \lambda_{j}(i):=\pi(i, j) / \rho_{j}, \rho_{i}(j):=\pi(i, j) / \lambda_{i}$.

\section{Evolution of Degree Distributions in Residual Graphs for Detailedly Represented Ensembles}

In this section, we derive expected degree distributions in residual graphs. We define original degree as the degree of nodes in the Tanner graph on which the code is defined. Residual degree of a node is defined as the degree of the node in the residual graph. Let $t$ denote time, which starts from zero and increases by $\Delta t:=1 / E$ for every decoding iteration, where $E$ is the number of edges contained in the Tanner graph on which the code is defined. Let $L_{k}(t), R_{s, \tilde{s}}(t)$ and $E_{k, s}(t)$ be the total number of edges connecting to variable nodes of residual degree $k$, connecting to check nodes of residual degree $\tilde{s}$ and original degree $s$, and connecting to variable nodes of original degree $k$ and check nodes of original degree $s$ at time $t$, respectively. For a given residual graph, we define variable degree distribution $l_{k}$, check degree distribution $r_{s, \tilde{s}}$ and joint degree distribution $e_{k, s}$ as $l_{k}:=L_{k} / E, r_{s, \tilde{s}}:=R_{s, \tilde{s}} / E$ and $e_{k, s}:=E_{k, s} / E$, respectively.

\subsection{Differential Equations Description}

From the iterations of PA, we can derive following differential equations.

Lemma 1: The differential equation for the joint degree distribution $e_{k, s}$, the variable degree distribution $l_{k}$ and the check degree distribution $r_{s, \tilde{s}}$ in residual graph, are given as

$$
\begin{aligned}
& \frac{\mathrm{d} e_{k, s}}{\mathrm{~d} t}=-h_{s} p_{k, s}-(k-1) q_{k, s} \sum_{j=2}^{\mathrm{r}_{\mathrm{m}}} h_{j} p_{k, j} \\
& \frac{\mathrm{d} l_{k}}{\mathrm{~d} t}=-k \sum_{j=2}^{\mathrm{r}_{\mathrm{m}}} h_{j} p_{k, j} \\
& \frac{\mathrm{d} r_{s, 1}}{\mathrm{~d} t}=-h_{s}+\frac{\left(r_{s, 2}-r_{s, 1}\right)}{\sum_{\tilde{s}^{\prime}=1}^{s} r_{s, \tilde{s}^{\prime}}} \sum_{i=2}^{l_{\mathrm{m}}}(i-1) q_{i, s} \sum_{j=2}^{\mathrm{r}_{\mathrm{m}}} h_{j} p_{i, j} \\
& \frac{\mathrm{d} r_{s, \tilde{s}}}{\mathrm{~d} t}=\tilde{s} \frac{\left(r_{s, \tilde{s}+1}-r_{s, \tilde{s}}\right)}{\sum_{\tilde{s}^{\prime}=1}^{s} r_{s, \tilde{s}^{\prime}}} \sum_{i=2}^{1_{\mathrm{m}}}(i-1) q_{i, s} \sum_{j=2}^{\mathrm{r}_{\mathrm{m}}} h_{j} p_{i, j},
\end{aligned}
$$

where

$$
h_{j}:=\frac{r_{j, 1}}{\sum_{j^{\prime}} r_{j^{\prime}, 1}}, p_{i, j}:=\frac{e_{i, j}}{\sum_{i^{\prime}} e_{i^{\prime}, j}}, q_{i, j}:=\frac{e_{i, j}}{\sum_{j^{\prime}} e_{i, j^{\prime}}} .
$$

\subsubsection{Proof of Eq. (1)}

A check node of residual degree one is chosen uniformly and removed on each iteration. Let $h_{j}$ be the probability that the chosen check node has original degree $j$. The probability $h_{j}$ is given by

$$
h_{j}=\frac{r_{j, 1}}{\sum_{j^{\prime}} r_{j^{\prime}, 1}} \text {. }
$$

Let $p_{i, j}$ be the probability that the edge connecting to the check node of original degree $j$ connects to a variable node of original degree $i$ at the other end. The probability $p_{i, j}$ is given by $p_{i, j}=e_{i, j} / \sum_{i^{\prime}} e_{i^{\prime}, j}$. Similarly, let $q_{i, j}$ be the probability that the edge connecting to the variable node of original degree $i$ connects to a check node of original degree $j$ at the other end. The probability $q_{i, j}$ is given by $q_{i, j}=e_{i, j} / \sum_{j^{\prime}} e_{i, j^{\prime}}$. Assume that we have a check node c of residual degree one and original degree $j$ and a variable node $\mathrm{v}$ of original degree $i$. Let $v_{i, j}\left(u_{2}, \ldots, u_{\mathrm{r}_{\mathrm{m}}}\right)$ be the probability that $i$ edges connecting to the variable node $\mathrm{v}$ connect to $u_{s}$ check nodes of original degree $s$, for $s \in\left\{2, \ldots, \mathrm{r}_{\mathrm{m}}\right\}$, including the check node $\mathrm{c}$, where $\sum_{s=2}^{\mathrm{r}_{\mathrm{m}}} u_{s}=i$. In the blocklength limit, the probability $v_{i, j}\left(u_{2}, \ldots, u_{\mathrm{r}_{\mathrm{m}}}\right)$ is given by

$$
\begin{aligned}
v_{i, j}\left(u_{2}, \ldots, u_{\mathrm{r}_{\mathrm{m}}}\right)= & \left(\begin{array}{c}
i-1 \\
u_{2}, \ldots, u_{j}-1, \ldots, u_{\mathrm{r}_{\mathrm{m}}}
\end{array}\right) \\
& \cdot q_{i, 2}^{u_{2}} \cdots q_{i, j}^{u_{j}-1} \cdots q_{i, \mathrm{r}_{\mathrm{m}}}^{u_{\mathrm{rm}}} .
\end{aligned}
$$

The probability generating function for $v_{i, j}\left(u_{2}, \ldots, u_{\mathrm{r}_{\mathrm{m}}}\right)$ is given by

$$
\begin{gathered}
\sum_{u_{2}, \ldots, u_{\mathrm{rm}}} v_{i, j}\left(u_{2}, \ldots, u_{\mathrm{r}_{\mathrm{m}}}\right) x_{2}^{u_{2}} \cdots x_{\mathrm{r}_{\mathrm{m}}}^{u_{\mathrm{r}_{\mathrm{m}}}} \\
=x_{j}\left(q_{i, 2} x_{2}+\cdots+q_{i, \mathrm{r}_{\mathrm{m}}} x_{\mathrm{r}_{\mathrm{m}}}\right)^{i-1} .
\end{gathered}
$$

Let $\hat{f}_{j}^{\left(e_{k, s}\right)}$ denote the expected change in the number of edges connecting to check nodes of original degree $s$ and variable nodes of original degree $k$, conditioned on the fact that a check node of original degree $j$ is removed. Taking the derivative of both sides of the Eq. (6) with respect to $x_{s}$ and 
$x_{j}=1$ for all $j$, we can write for $j \neq s$

$$
\begin{aligned}
\hat{f}_{j}^{\left(e_{k, s}\right)} & =-\sum_{u_{2}, \ldots, u_{\mathrm{rm}}} p_{k, j} v_{k, j}\left(u_{2}, \ldots, u_{\mathrm{r}_{\mathrm{m}}}\right) u_{s} \\
& =-(k-1) p_{k, j} q_{k, s} .
\end{aligned}
$$

Similarly, for $j=s$, we can write

$$
\begin{aligned}
\hat{f}_{s}^{\left(e_{k, s}\right)} & =-\sum_{u_{2}, \ldots, u_{\mathrm{rm}}} p_{k, s} v_{k, s}\left(u_{2}, \ldots, u_{\mathrm{r}_{\mathrm{m}}}\right) u_{s} \\
& =-p_{k, s}\left(1+(k-1) q_{k, s}\right) .
\end{aligned}
$$

Let $\hat{f}^{\left(e_{k, s}\right)}$ denote the expected change in the number of edges connecting to check nodes of original degree $s$ and variable nodes of original degree $k$. From Eqs. (5), (7) and (8), we see that

$$
\hat{f}^{\left(e_{k, s}\right)}=\sum_{j=2}^{\mathrm{r}_{\mathrm{m}}} h_{j} \hat{f}_{j}^{\left(e_{k, s}\right)}=-h_{s} p_{k, s}-(k-1) q_{k, s} \sum_{j=2}^{\mathrm{r}_{\mathrm{m}}} h_{j} p_{k, j} .
$$

As $E \rightarrow \infty, \Delta t$ tends to 0 . Note that $\hat{f}^{\left(e_{k, s}\right)}=E_{k, s}(t+\Delta t)-$ $E_{k, s}(t)=\left\{e_{k, s}(t+\Delta t)-e_{k, s}(t)\right\} / \Delta t=\frac{\mathrm{d} e_{k, s}}{\mathrm{~d} t}$. Hence, we obtain Eq. (1).

\subsubsection{Proof of Eq. (2)}

Let $\hat{f}_{j}^{\left(l_{k}\right)}$ denote the expected change in the number of the edges connecting to variable nodes of residual degree $k$ conditioned on the fact that a check node of original degree $j$ is removed. We can write

$$
\hat{f}_{j}^{\left(l_{k}\right)}=-k p_{k, j}
$$

Let $\hat{f}^{\left(l_{k}\right)}$ denote the expected change in the number of edges connecting to variable nodes of residual degree $k$. From Eqs. (5) and (9), we see that

$$
\hat{f}^{\left(l_{k}\right)}=\sum_{j=2}^{\mathrm{r}_{\mathrm{m}}} h_{j} \hat{f}_{j}^{\left(l_{k}\right)}=-k \sum_{j=2}^{\mathrm{r}_{\mathrm{m}}} h_{j} p_{k, j} .
$$

Similarly, note that $\hat{f}^{\left(l_{k}\right)}=\frac{\mathrm{d} l_{k}}{\mathrm{~d} t}$. Hence we obtain Eq. (2).

\subsubsection{Proof of Eqs. (3) and (4)}

Let $q_{i, s, \tilde{s}}$ be the probability that the edge connecting to the variable node of original degree $i$ has a check node of original degree $s$ and residual degree $\tilde{s}$. The probability $q_{i, s, \tilde{s}}$ is given by $q_{i, s, \tilde{s}}=q_{i, s} r_{s, \tilde{s}} / \sum_{\tilde{s}^{\prime}} r_{s, \tilde{s}^{\prime}}$. Assume that we have a check node $\mathrm{c}$ of residual degree one and original degree $j$ and a variable node $\mathrm{v}$ of original degree $i$. Let $w_{i, j}\left(u_{2,1}, \ldots, u_{\mathrm{r}_{\mathrm{m}}, \mathrm{r}_{\mathrm{m}}}\right)$ be the probability that $i$ edges connecting to the variable node $\mathrm{v}$ connect to $u_{s, \tilde{s}}$ check nodes of original degree $s$ and residual degree $\tilde{s}$, for $s \in\left\{2, \ldots, \mathrm{r}_{\mathrm{m}}\right\}$ and $\tilde{s} \in\{1, \ldots, s\}$, including the check node c, where $\sum_{s=2}^{\mathrm{r}_{\mathrm{m}}} \sum_{\tilde{s}=1}^{s} u_{s, \tilde{s}}=i$. In the blocklength limit, the probability $w_{i, j}\left(u_{2,1}, \ldots, u_{\mathrm{r}_{\mathrm{m}}, \mathrm{r}_{\mathrm{m}}}\right)$ is given by

$$
\begin{aligned}
w_{i, j} & \left(u_{2,1}, \ldots, u_{\mathrm{r}_{\mathrm{m}}, \mathrm{r}_{\mathrm{m}}}\right) \\
= & \left(\begin{array}{c}
i-1 \\
u_{2,1}, \ldots, u_{j, 1}-1, \ldots, u_{\mathrm{r}_{\mathrm{m}}, \mathrm{r}_{\mathrm{m}}}
\end{array}\right) \\
& \cdot q_{i, 2,1}^{u_{2,1}} \cdots q_{i, j, 1}^{u_{j, 1}-1} \cdots q_{i, \mathrm{r}_{\mathrm{m}}, \mathrm{r}_{\mathrm{m}}}^{u_{\mathrm{r} \mathrm{m}}} .
\end{aligned}
$$

From this equation, the probability generating function for $w_{i, j}\left(x_{2,1}, \ldots, x_{\mathrm{r}_{\mathrm{m}}, \mathrm{r}_{\mathrm{m}}}\right)$ is given by

$$
\begin{aligned}
& \sum_{u_{2,1}, \ldots, u_{\mathrm{r}}, \mathrm{r}_{\mathrm{m}}} w_{i, j}\left(u_{2,1}, \ldots, u_{\mathrm{r}_{\mathrm{m}}, \mathrm{r}_{\mathrm{m}}}\right) x_{2,1}^{u_{2,1}} \cdots x_{\mathrm{r}_{\mathrm{m}}, \mathrm{r}_{\mathrm{m}}}^{u_{\mathrm{rm}}, \mathrm{r}_{\mathrm{m}}} \\
& =x_{j, 1}\left(q_{i, 2,1} x_{2,1}+\cdots+q_{i, \mathrm{r}_{\mathrm{m}}, \mathrm{r}_{\mathrm{m}}} x_{\mathrm{r}_{\mathrm{m}}, \mathrm{r}_{\mathrm{m}}}\right)^{i-1} .
\end{aligned}
$$

We assume that the edge connecting to a check node of original degree $s$ and residual degree $\tilde{s}$ is removed. The decrease in the number of edges connecting to check nodes of original degree $s$ and residual degree $\tilde{s}$ is $\tilde{s}$. On the other hand, the increase in the number of edges connecting to check nodes of original degree $s$ and residual degree $\tilde{s}-1$ is $\tilde{s}-1$. Let $\hat{f}_{j}^{\left(r_{s, \tilde{s}}\right)}$ denote the expected change in the number of edges connecting to check nodes of original degree $s$ and residual degree $\tilde{s}$, conditioned on the fact that a check node of original degree $j$ is removed. Using Eq. (10), for $j \neq s$ or $\tilde{s} \neq 1$, we can write

$$
\begin{aligned}
\hat{f}_{j}^{\left(r_{s, \tilde{s}}\right)}= & \tilde{s} \sum_{i=2}^{1_{\mathrm{m}}} p_{i, j} \sum_{u_{2,1}, \ldots, u_{\mathrm{r}, \mathrm{rm}}} \\
& w_{i, j}\left(u_{2,1}, \ldots, u_{\mathrm{r}_{\mathrm{m}}, \mathrm{r}_{\mathrm{m}}}\right)\left(u_{s, \tilde{s}+1}-u_{s, \tilde{s}}\right) \\
= & \tilde{s} \sum_{i=2}^{1_{\mathrm{m}}} p_{i, j}(i-1)\left(q_{i, s, \tilde{s}+1}-q_{i, s, \tilde{s}}\right) .
\end{aligned}
$$

Similarly, for $j=s$ and $\tilde{s}=1$, we can write

$$
\begin{aligned}
\hat{f}_{s}^{\left(r_{s, 1}\right)}= & \sum_{i=2}^{1_{\mathrm{m}}} p_{i, s} \sum_{u_{2,1}, \ldots, u_{\mathrm{r}_{\mathrm{m}}, \mathrm{r}_{\mathrm{m}}}} \\
& w_{i, s}\left(u_{2,1}, \ldots, u_{\mathrm{r}_{\mathrm{m}}, \mathrm{r}_{\mathrm{m}}}\right)\left(u_{s, 2}-u_{s, 1}\right) \\
= & -1+\sum_{i=2}^{1_{\mathrm{m}}} p_{i, s}(i-1)\left(q_{i, s, 2}-q_{i, s, 1}\right) .
\end{aligned}
$$

Let $\hat{f}^{\left(r_{s, \tilde{s}}\right)}$ denote the expected change in the number of edges connecting to check nodes of original degree $s$ and residual degree $\tilde{s}$. From Eqs. (5), (11) and (12), for $\tilde{s} \geq 2$, we see that

$$
\begin{aligned}
\hat{f}^{\left(r_{s, \tilde{s}}\right)} & =\sum_{j=2}^{\mathrm{r}_{\mathrm{m}}} h_{j} \hat{f}_{j}^{\left(r_{s, \tilde{s}}\right)} \\
& =\tilde{s} \frac{\left(r_{s, \tilde{s}+1}-r_{s, \tilde{s}}\right)}{\sum_{\tilde{s}} r_{s, \tilde{s}}} \sum_{i=2}^{1_{\mathrm{m}}}(i-1) q_{i, s} \sum_{j=2}^{\mathrm{r}_{\mathrm{m}}} h_{j} p_{i, j} .
\end{aligned}
$$

Similarly, for $\tilde{s}=1$, we see that

$$
\begin{aligned}
\hat{f}^{\left(r_{s, 1}\right)} & =\sum_{j=2}^{r_{\mathrm{m}}} h_{j} \hat{f}_{j}^{\left(r_{s, 1}\right)} \\
& =-h_{s}+\frac{\left(r_{s, 2}-r_{s, 1}\right)}{\sum_{\tilde{s}} r_{s, \tilde{s}}} \sum_{i=2}^{1_{\mathrm{m}}}(i-1) q_{i, s} \sum_{j=2}^{\mathrm{r}_{\mathrm{m}}} h_{j} p_{i, j} .
\end{aligned}
$$


Note that $\hat{f}^{\left(r_{s, \tilde{s}}\right)}=\frac{\mathrm{d} r_{s, \tilde{s}}}{\mathrm{~d} t}$. Hence, we obtain Eqs. (3) and (4).

\subsection{Evolution of Degree Distributions in Residual Graphs}

Unfortunately, it is difficult to solve differential equations in Lemma 1. However, these differential equations are easily solved by parameters $\mathbf{y}=\left(y_{2}, \ldots, y_{\mathrm{r}_{\mathrm{m}}}\right)$. The parameters $\mathbf{y}$ are defined such that

$$
\frac{\mathrm{d} y_{j}}{\mathrm{~d} t}=-\frac{y_{j}}{\sum_{i^{\prime}=2}^{1_{\mathrm{m}}} e_{i^{\prime}, j}} \frac{r_{j, 1}}{\sum_{j^{\prime}} r_{j^{\prime}, 1}}
$$

and $y_{j}=1$ when $t=0$.

Theorem 1: Using parameters $\mathbf{y}=\left(y_{2}, \ldots, y_{\mathrm{r}_{\mathrm{m}}}\right)$, the joint degree distribution $e_{k, s}$, the variable degree distribution $l_{k}$ and the check degree distribution $r_{s, \tilde{s}}$ in residual graph, are given as

$$
\begin{aligned}
& r_{s, 1}= \epsilon \sum_{i=2}^{1_{\mathrm{m}}} \pi(i, s)\left(\sum_{j=2}^{\mathrm{r}_{\mathrm{m}}} \rho_{i}(j) y_{j}\right)^{i-1}\left[y_{s}-1\right. \\
&\left.+\left\{1-\epsilon \sum_{i=2}^{1_{\mathrm{m}}} \lambda_{s}(i)\left(\sum_{j=2}^{\mathrm{r}_{\mathrm{m}}} \rho_{i}(j) y_{j}\right)^{i-1}\right\}^{s-1}\right] \\
& r_{s, \tilde{s}=} \rho_{s}\left(\begin{array}{c}
s-1 \\
\tilde{s}-1
\end{array}\right)\left\{\epsilon \sum_{i=2}^{1_{\mathrm{m}}} \lambda_{s}(i)\left(\sum_{j=2}^{\mathrm{r}_{\mathrm{m}}} \rho_{i}(j) y_{j}\right)^{i-1}\right\}^{\tilde{s}} \\
& \cdot\left\{1-\epsilon \sum_{i=2}^{1_{\mathrm{m}}} \lambda_{s}(i)\left(\sum_{j=2}^{\mathrm{r}_{\mathrm{m}}} \rho_{i}(j) y_{j}\right\}^{s-1}\right. \\
& l_{k}=\epsilon \lambda_{k}\left(\sum_{j=2}^{\mathrm{r}_{\mathrm{m}}} \rho_{k}(j) y_{j}\right)^{k} \\
& e_{k, s}= \epsilon \pi(k, s) y_{s}\left(\sum_{j=2}^{\mathrm{r}_{\mathrm{m}}} \rho_{k}(j) y_{j}\right)^{k-1},
\end{aligned}
$$

for $k \in\left\{2, \ldots, 1_{\mathrm{m}}\right\}, s \in\left\{2, \ldots, \mathrm{r}_{\mathrm{m}}\right\}$ and $\tilde{s} \in\{2, \ldots, s\}$.

The proof of Theorem 1 is given in Appendix.

Example 1: Using Eqs. (14) and (17) for Eq. (13), we can write

$$
\frac{\mathrm{d} y_{j}}{\mathrm{~d} t}=-\frac{y_{j}-1+\left(1-\epsilon \sum_{i} \lambda_{j}(i) Y_{i}^{i-1}\right)^{j-1}}{\sum_{j^{\prime}} r_{j^{\prime}, 1}},
$$

where $\sum_{j} r_{j, 1}=\epsilon \sum_{j} \sum_{i^{\prime}} \pi\left(i^{\prime}, j\right) Y_{i^{\prime}}^{i^{\prime}-1}\left\{y_{j}-1+(1-\right.$ $\left.\left.\epsilon \sum_{i} \lambda_{j}(i) Y_{i}^{i-1}\right)^{j-1}\right\}$ and $Y_{i}=\sum_{j=2}^{\mathrm{r}_{\mathrm{m}}} \rho_{i}(j) y_{j}$. Solving Eq. (18) by numerical calculations, we have $\mathbf{y}$ as a function of $t$. Using $\mathbf{y}$, from Theorem 1, we have degree distributions in residual graphs at time $t$. If decoding succeeds, the number of iteration of PA equals to the number of variable nodes in residual graph at $t=0$, since each iteration of PA removes a variable node. The decoding halts at $t=t_{\max }:=\epsilon \sum_{i} \frac{E \lambda_{i}}{i} \Delta t=\epsilon \sum_{i} \frac{\lambda_{i}}{i}$ for successful decoding. Figure 1 shows the evolution of the degree distributions $r_{5,1}(t)$ and $r_{10,1}(t)$ in residual graphs for $\pi(3,5)=0.12$,

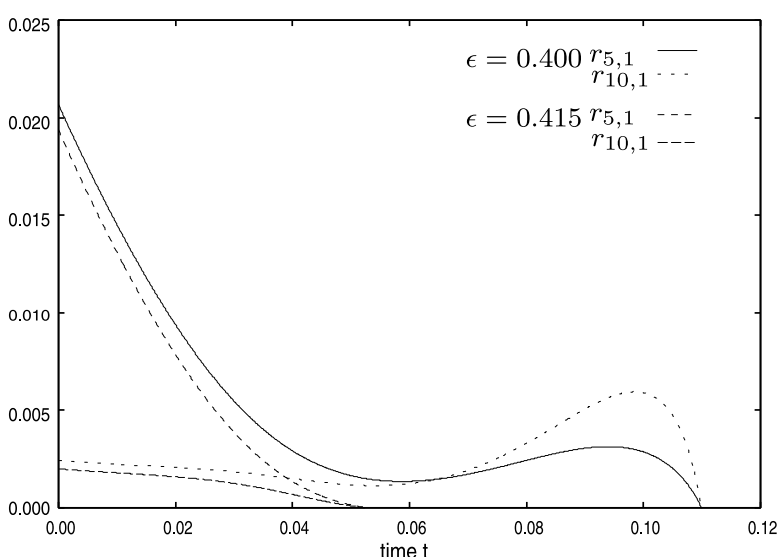

Fig. 1 Evolution of degree distributions $r_{5,1}$ and $r_{10,1}$ in residual graphs. $\pi(3,5)=0.12, \pi(3,10)=0.18, \pi(4,5)=0.28, \pi(4,10)=0.42$.

$\pi(3,10)=0.18, \pi(4,5)=0.28, \pi(4,10)=0.42, t_{\max }=0.11$. Since the curves of $\epsilon=0.400$ reach to $t_{\max }$ in Fig. 1, we see that decoding succeeds. On the other hand, the curves of $\epsilon=0.415$ touch the zero line at $t<t_{\max }$ and the decoding fails i.e. the residual graph has no check node of residual degree one.

\section{Graphical Determination of Successful Decoding}

In this section, we see $r_{s, 1}$ in Eq. (14) as a function of $\mathbf{y}$. The following theorem shows a graphical determination of successful decoding from the contour of $\min _{s} r_{s, 1}(\mathbf{y})$.

Theorem 2: For detailedly represented ensembles and $\epsilon \epsilon$ $(0,1)$, a path from the point $(1,1, \ldots, 1)$ to $(0,0, \ldots, 0)$ exists in the set $\left\{\mathbf{y} \mid \min _{s} r_{s, 1}(\epsilon, \mathbf{y})>0, \mathbf{y} \in[0,1]^{|\mathbb{D}|}\right\}$ if and only if the PA is successful.

The proof of Theorem 2 is given in Sects. 4.1 and 4.2. An example of Theorem 2 is given in the following.

Example 2 (Two Different Check Degrees): Let $y_{m}^{(\ell)}$ also represent the fraction of erased messages sent from the check nodes of degree $m$ to variable nodes at the $\ell$-th iteration of $\mathrm{BP}$ which is equivalent with $\mathrm{PA}$ in decoding results. (It is described in Sect.4.1). Define $\mathbf{y}^{(\ell)}:=$ $\left(y_{2}^{(\ell)}, y_{3}^{(\ell)}, \ldots, y_{\mathrm{r}_{\mathrm{m}}}^{(\ell)}\right)$. If $\mathbf{y}^{(\ell)}=(0,0, \ldots, 0)$ for some $\ell$, then $\mathrm{BP}$ decoding is successful. We define path of density evolution as the path made from the line segments bounded by the point $\mathbf{y}^{(\ell)}$ and $\mathbf{y}^{(\ell+1)}$ for all $\ell \geq 0$. The set $\{\mathbf{y} \mid$ $\left.\min \left\{r_{5,1}(\mathbf{y}), r_{10,1}(\mathbf{y})\right\}>0, \mathbf{y} \in[0,1]^{2}\right\}$ is the region inside of curves labeled 0.00 in Fig. 2 and Fig. 3. In Fig. 2, the point $(1,1)$ and $(0,0)$ are connected by the path of density evolution and decoding is successful. On the other hand, in Fig. 3, there does not exists any path from the point $(1,1)$ and $(0,0)$ in the set $\left\{\mathbf{y} \mid \min \left\{r_{5,1}(\mathbf{y}), r_{10,1}(\mathbf{y})\right\}>0, \mathbf{y} \in[0,1]^{2}\right\}$ and the decoding is not successful.

\subsection{Monotonicity}

For $s \in\left\{2, \ldots, \mathrm{r}_{\mathrm{m}}\right\}$, functions $f_{s}(\epsilon, \mathbf{y})$ are defined as 


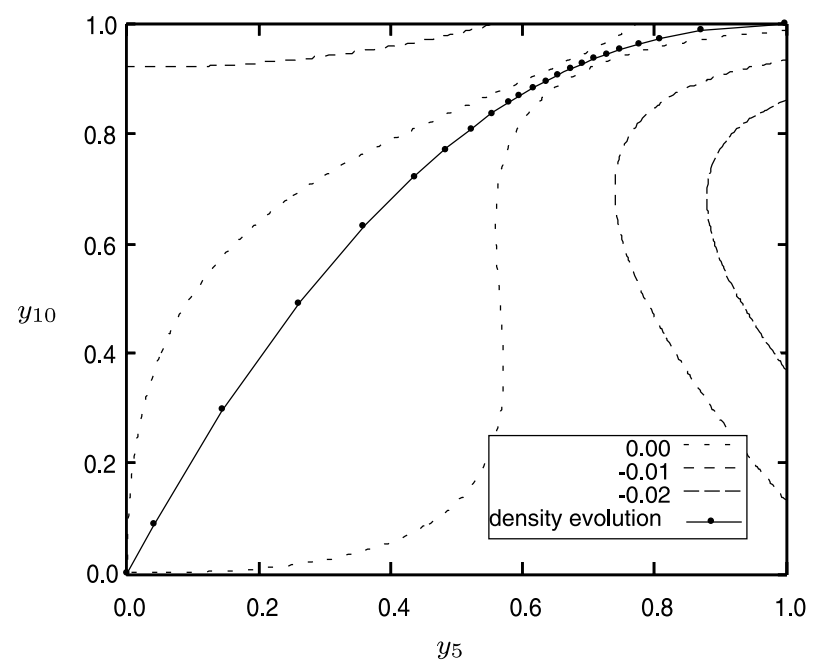

Fig. 2 The contour of $\min \left\{r_{5,1}, r_{10,1}\right\}$ and the path of density evolution (d.e.). $\pi(3,5)=0.12, \pi(3,10)=0.18, \pi(4,5)=0.28, \pi(4,10)=0.42$, $\epsilon=0.40$.

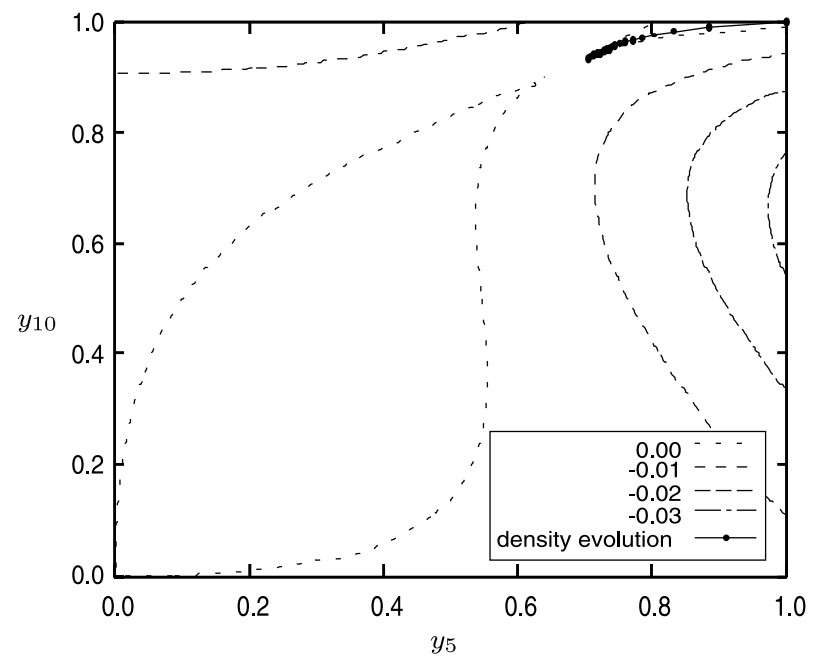

Fig. 3 The contour of $\min \left\{r_{5,1}, r_{10,1}\right\}$ and path of density evolution. $\pi(3,5)=0.12, \pi(3,10)=0.18, \pi(4,5)=0.28, \pi(4,10)=0.42, \epsilon=0.415$.

$$
f_{s}(\epsilon, \mathbf{y}):=1-\left\{1-\epsilon \sum_{i=2}^{1_{\mathrm{m}}} \lambda_{s}(i)\left(\sum_{j=2}^{\mathrm{r}_{\mathrm{m}}} \rho_{i}(j) y_{j}\right)^{i-1}\right\}^{s-1} .
$$

These functions are called density evolution equations. We start with $\mathbf{y}^{(0)}=(1, \ldots, 1)$ and apply the recursions $y_{s}^{(\ell+1)}=$ $f_{s}\left(\epsilon, \mathbf{y}^{(\ell)}\right)$. We denote $\mathbf{y}^{(\ell+1)}<\mathbf{y}^{(\ell)}$ if $y_{s}^{(\ell+1)}<y_{s}^{(\ell)}$ for all $s \in$ $\left\{2, \ldots, \mathrm{r}_{\mathrm{m}}\right\}$. A point $\mathbf{p}$ is called a fixed point if $p_{s}=f_{s}(\epsilon, \mathbf{p})$ for all $s \in\left\{2, \ldots, \mathrm{r}_{\mathrm{m}}\right\}$. To prove Theorem 2 , we use the following lemmas.

Lemma 2: For $\epsilon \in(0,1)$ and $\mathbf{y} \neq(0, \ldots, 0), f_{s}(\epsilon, \mathbf{y})$ is increasing in $y_{k}$ for all $s, k \in\left\{2, \ldots, \mathrm{r}_{\mathrm{m}}\right\}$.

Lemma 3: For $\epsilon \in(0,1)$, the sequence $\left\{\mathbf{y}^{(\ell)}\right\}_{\ell \geq 0}$ converges to a fixed point and $\mathbf{y}^{(\ell+1)}<\mathbf{y}^{(\ell)}$ for all $\ell \geq 0$.
Proof of Lemma 2: For $k, s \in\left\{2, \ldots, \mathrm{r}_{\mathrm{m}}\right\}, \epsilon \in(0,1)$ and $\mathbf{y} \neq(0, \ldots, 0)$, the partial derivative of Eq. (19) with respect to $y_{k}$ is

$$
\begin{gathered}
\frac{\partial f_{s}(\epsilon, \mathbf{y})}{\partial y_{k}}=(s-1) \epsilon \sum_{i=2}^{\mathrm{l}_{\mathrm{m}}} \lambda_{s}(i)(i-1) \rho_{i}(k)\left(\sum_{j=2}^{\mathrm{r}_{\mathrm{m}}} \rho_{i}(j) y_{j}\right)^{i-2} \\
\cdot\left\{1-\epsilon \sum_{i=2}^{\mathrm{l}_{\mathrm{m}}} \lambda_{s}(i)\left(\sum_{j=2}^{\mathrm{r}_{\mathrm{m}}} \rho_{i}(j) y_{j}\right)^{i-1}\right\}^{s-2}>0
\end{gathered}
$$

and we conclude that $f_{s}(\epsilon, \mathbf{y})$ is increasing in $y_{k}$.

Proof of Lemma 3: Let $\epsilon \in(0,1)$. It holds that $\mathbf{y}^{(1)}<$ $\mathbf{y}^{(0)}$ since $y_{s}^{(1)}=f_{s}\left(\epsilon, \mathbf{y}^{(0)}\right)=1-(1-\epsilon)^{s-1}<1=y_{s}^{(0)}$ for $s \in\left\{2, \cdots, \mathrm{r}_{\mathrm{m}}\right\}$. From Lemma 2, if $\mathbf{y}^{(\ell+1)}<\mathbf{y}^{(\ell)}$ then $y_{s}^{(\ell+2)}=f_{s}\left(\epsilon, \mathbf{y}^{(\ell+1)}\right)<f_{s}\left(\epsilon, \mathbf{y}^{(\ell)}\right)=y_{s}^{(\ell+1)}$ for $s \in\left\{2, \cdots, \mathrm{r}_{\mathrm{m}}\right\}$. This shows $\mathbf{y}^{(\ell+2)}<\mathbf{y}^{(\ell+1)}$. Hence, $\mathbf{y}^{(\ell+1)}<\mathbf{y}^{(\ell)}$ for all $\ell \geq 0$.

Let $\mathbf{p}$ be a fixed point. It can be shown that from Lemma 2, if $\mathbf{p} \leq \mathbf{y}^{(\ell)}$ then $p_{s}=f_{s}(\epsilon, \mathbf{p}) \leq f_{s}\left(\epsilon, \mathbf{y}^{(\ell)}\right)=y_{s}^{(\ell+1)}$. This shows $\mathbf{p} \leq \mathbf{y}^{(\ell+1)}$. Therefore, the sequence $\left\{\mathbf{y}^{(\ell)}\right\}_{\ell \geq 0}$ converges. Let $\mathbf{y}^{(\infty)}:=\lim _{\ell \rightarrow \infty} \mathbf{y}^{(\ell)}$. Since the sequence converges, $y_{s}^{(\infty)}=f_{s}\left(\epsilon, \mathbf{y}^{(\infty)}\right)$ for all $s$. This shows $\mathbf{y}^{(\infty)}$ is a fixed point. Hence, the sequence $\left\{\mathbf{y}^{(\ell)}\right\}_{\ell \geq 0}$ converges to the fixed point.

\subsection{Proof of Theorem 2}

Define $\mathcal{R}:=\left\{\mathbf{y} \mid \min _{s} r_{s, 1}(\epsilon, \mathbf{y})>0, \mathbf{y} \in[0,1]^{|\mathbb{D}|}\right\}$. From Eqs. (14) and (19), it holds for $\epsilon \in(0,1)$

$$
y_{s}>f_{s}(\epsilon, \mathbf{y}) \text { iff } r_{s, 1}(\epsilon, \mathbf{y})>0 .
$$

From Lemma 2 and Lemma 3, it can be shown $y_{s}^{(\ell+1)}=$ $f_{s}\left(\epsilon, \mathbf{y}^{(\ell)}\right)>f_{s}\left(\epsilon, \mathbf{y}^{(\ell+1)}\right)$ for all $s \in\left\{2, \ldots, \mathrm{r}_{\mathrm{m}}\right\}$. From Eq. (20), $r_{s, 1}\left(\epsilon, \mathbf{y}^{(\ell)}\right)>0$ for $\ell \geq 1$. From Eq. (14), $r_{s, 1}\left(\epsilon, \mathbf{y}^{(0)}\right)>0$. Therefore, we obtain $\mathbf{y}^{(\ell)} \in \mathcal{R}$ for $\ell \geq 0$. Let $\theta \in(0,1)$. Define $\mathbf{a}(\theta):=\theta \mathbf{y}^{(\ell)}+(1-\theta) \mathbf{y}^{(\ell+1)}$. Since $\mathbf{y}^{(\ell+1)}<\mathbf{a}(\theta)<\mathbf{y}^{(\ell)}$, we obtain $f_{s}(\epsilon, \mathbf{a}(\theta))<f_{s}\left(\epsilon, \mathbf{y}^{(\ell)}\right)=$ $y_{s}^{(\ell+1)}$ for all $s \in\left\{2, \cdots, \mathrm{r}_{\mathrm{m}}\right\}$ by Lemma 2. This shows $f_{s}(\epsilon, \mathbf{a}(\theta))<a_{s}(\theta)$ for all $s$. From Eq. $(20), \mathbf{a}(\theta) \in \mathcal{R}$. Define $\mathcal{L}^{(\ell)}:=\left\{\theta \mathbf{y}^{(\ell)}+(1-\theta) \mathbf{y}^{(\ell+1)} \mid \theta \in[0,1]\right\}$. We conclude that $\mathcal{L}^{(\ell)} \subset \mathcal{R}$ for all $\ell \geq 0$. If decoding is successful, then $\mathbf{y}^{(\infty)}=(0,0, \ldots, 0)$. There exists a path from the point $(1, \ldots, 1)$ to $(0, \ldots, 0)$ by line segments $\mathcal{L}^{(\ell)}$. Since $\mathcal{L}^{(\ell)} \subset \mathcal{R}$, this path is in the set $\mathcal{R}$.

If decoding is not successful, then some fixed points, represented by $\mathbf{p} \in(0,1]^{|\mathbb{D}|}$, exist. Define $\mathcal{P}_{s}:=\left\{\mathbf{y} \mid y_{i} \in\right.$ $\left.\left[p_{i}, 1\right], \forall i \in\left\{2, \ldots, \mathrm{r}_{\mathrm{m}}\right\} /\{s\}, y_{s}=p_{s}\right\}$ for $s \in\left\{2, \ldots, \mathrm{r}_{\mathrm{m}}\right\}$. Let $\mathbf{b}_{s} \in \mathcal{P}_{s}$. Since $\mathbf{p} \leq \mathbf{b}_{s}$, it holds that $f_{s}(\epsilon, \mathbf{p}) \leq f_{s}\left(\epsilon, \mathbf{b}_{s}\right)$ by Lemma 2. Since $p_{s}-f_{s}(\epsilon, \mathbf{p}) \geq p_{s}-f_{s}\left(\epsilon, \mathbf{b}_{s}\right)$, we obtain $0=$ $r_{s, 1}(\epsilon, \mathbf{p}) \geq r_{s, 1}\left(\epsilon, \mathbf{b}_{s}\right)$ from $\operatorname{Eq}(20)$. Therefore, $\mathcal{P}_{s} \cap \mathcal{R}=\emptyset$. Every path from the point $(1, \ldots, 1)$ to $(0, \ldots, 0)$ includes a point in a set $\mathcal{P}_{s}$. Hence, there does not exists any path from the point $(1, \ldots, 1)$ to $(0, \ldots, 0)$ in the set $\mathcal{R}$. 


\section{Conclusion and Future Work}

In this paper, we derive detailed evolution of degree distributions in residual graphs using joint degree distribution. From this results, we can derive the average of the number of check nodes of degree one in residual graph. Moreover, we give a graphical determination of successful decoding.

As a future work, we will derive the variance of the number of check nodes of degree one in residual graphs, and will show finite-length scaling [5] for detailedly represented ensembles. Moreover we will optimize finite-length LDPC codes for detailedly represented ensembles.

\section{References}

[1] R.G. Gallager, Low-Density Parity-check Codes, MIT Press, 1963.

[2] M. Luby, M. Mitzenmacher, A. Shokrollahi, and D. Spielman, "Improved low-density parity-check codes using irregular graphs," IEEE Trans. Inf. Theory, vol.47, no.2, pp.585-598, Feb. 2001.

[3] K. Kasai, T. Shibuya, and K. Sakaniwa, "Detailedly represented irregular low-density parity-check codes," IEICE Trans. Fundamentals, vol.E86-A, no.10, pp.2435-2444, Oct. 2003.

[4] M. Luby, M. Mitzenmacher, A. Shokrollahi, D.A. Spielman, and V. Stemann, "Practical loss-resilient codes," Proc. 29th Annual ACM Symposium on Theory of Computing, pp.150-159, 1997.

[5] A. Amraoui, Asymptotic and finite-length optimization of LDPC codes, Ph.D. Thesis, EPFL, June 2006.

[6] T.J. Richardson, M.A. Shokrollahi, and R.L. Urbanke, "Design of capacity-approaching irregular low-density parity-check codes," IEEE Trans. Inf. Theory, vol.47, no.2, pp.619-637, 2001.

[7] T. Richardson and R. Urbanke, Modern Coding Theory, Cambridge University Press, 2008.

\section{Appendix: Proof of Theorem1}

For $j \in\left\{2, \ldots, \mathrm{r}_{\mathrm{m}}\right\}$ and $k \in\left\{2, \ldots, \mathrm{l}_{\mathrm{m}}\right\}$, define the variables $y_{j, k}$ such that

$$
\frac{\rho_{k}(j)}{\sum_{j=2}^{\mathrm{m}_{\mathrm{m}}} \rho_{k}(j) y_{j, k}} \mathrm{~d} y_{j, k}:=-\frac{h_{j} p_{k, j}}{l_{k}} \mathrm{~d} t,
$$

where $y_{j, k}=1$ when $t=0$ and they are called initial conditions. We define $\mathbf{y}_{k}:=\left(y_{2, k}, \ldots, y_{\mathrm{r}_{\mathrm{m}}, k}\right)$.

\section{A.1 Proof of Eqs. (16) and (17)}

Using Eq. (A· 1) for Eq. (2), we can write

$$
\begin{aligned}
& \mathrm{d} l_{k}=-k \sum_{j^{\prime}=2}^{\mathrm{r}_{\mathrm{m}}} h_{j^{\prime}} p_{k, j^{\prime}} \mathrm{d} t \\
& \mathrm{~d} l_{k}\left(\mathbf{y}_{k}\right)=k \frac{\sum_{j^{\prime}=2}^{\mathrm{r}_{\mathrm{m}}} \rho_{k}\left(j^{\prime}\right) \mathrm{d} y_{j^{\prime}, k}}{\sum_{j=2}^{\mathrm{r}_{\mathrm{m}}} \rho_{k}(j) y_{j, k}} l_{k}\left(\mathbf{y}_{k}\right) .
\end{aligned}
$$

We define the substitution $Y_{k}=\sum_{j=2}^{\mathrm{r}_{\mathrm{m}}} \rho_{k}(j) y_{j, k}$ which gives $\mathrm{d} Y_{k}=\sum_{j^{\prime}=2}^{\mathrm{r}_{\mathrm{m}}} \rho_{k}\left(j^{\prime}\right) \mathrm{d} y_{j^{\prime}, k}$. These transform Eq. (A. 2) to

$$
\mathrm{d} l_{k}\left(\mathbf{y}_{k}\right)=k \frac{\mathrm{d} Y_{k}}{Y_{k}} l_{k}\left(\mathbf{y}_{k}\right) .
$$

The solution is given by

$$
l_{k}\left(\mathbf{y}_{k}\right)=C\left(Y_{k}\right)^{k}=C\left(\sum_{j=2}^{\mathrm{r}_{\mathrm{m}}} \rho_{k}(j) y_{j, k}\right)^{k}
$$

for a constant $C$ to be determined from the initial condition. From initial conditions, we see that $l_{k}(t=0)=C=\epsilon \lambda_{k}$, since the erasure probability of each variable node is $\epsilon$. Hence, we obtain

$$
l_{k}\left(\mathbf{y}_{k}\right)=\epsilon \lambda_{k}\left(\sum_{j=2}^{\mathrm{r}_{\mathrm{m}}} \rho_{k}(j) y_{j, k}\right)^{k} .
$$

Using Eqs. (A. 1) and (A. 3) for Eq. (1), we can write

$$
\begin{aligned}
\mathrm{d} e_{k, s}= & -h_{s} p_{k, s} \mathrm{~d} t-(k-1) q_{k, s} \sum_{j^{\prime}=2}^{\mathrm{r}_{\mathrm{m}}} h_{j^{\prime}} p_{k, j^{\prime}} \mathrm{d} t \\
= & \epsilon \pi(k, s)\left(\sum_{j=2}^{\mathrm{r}_{\mathrm{m}}} \rho_{k}(j) y_{j, k}\right)^{k-1} \mathrm{~d} y_{s, k} \\
& +(k-1) e_{k, s}\left(\mathbf{y}_{k}\right) \frac{\sum_{j^{\prime}=2}^{\mathrm{r}_{\mathrm{m}}} \rho_{k}\left(j^{\prime}\right) \mathrm{d} y_{j^{\prime}, k}}{\sum_{j=2}^{\mathrm{r}_{\mathrm{m}}} \rho_{k}(j) y_{j, k}}
\end{aligned}
$$

where we use $q_{k, s}=e_{k, s} / l_{k}$. We can make this total differential equation exact, as following,

$$
\begin{aligned}
0=\mathrm{d} f= & -\frac{\mathrm{d} e_{k, s}}{\left(\sum_{j=2}^{\mathrm{r}_{\mathrm{m}}} \rho_{k}(j) y_{j, k}\right)^{k-1}}+\epsilon \pi(k, s) \mathrm{d} y_{s, k} \\
& +(k-1) e_{k, s} \frac{\sum_{j^{\prime}=2}^{\mathrm{r}_{\mathrm{m}}} \rho_{k}\left(j^{\prime}\right) \mathrm{d} y_{j^{\prime}, k}}{\left(\sum_{j=2}^{\mathrm{r}_{\mathrm{m}}} \rho_{k}(j) y_{j, k}\right)^{k}} .
\end{aligned}
$$

We assume that the variables $y_{s, k}\left(s \in\left\{2, \ldots, \mathrm{r}_{\mathrm{m}}\right\}, k \in\right.$ $\left.\left\{2, \ldots, 1_{\mathrm{m}}\right\}\right)$ are constants and the solution is given by

$$
f=-\frac{e_{k, s}}{\left(\sum_{j=2}^{\mathrm{r}_{\mathrm{m}}} \rho_{k}(j) y_{j, k}\right)^{k-1}}+C\left(\mathbf{y}_{k}\right),
$$

for the function $C\left(\mathbf{y}_{k}\right)$ to be determined from Eq. (A.4). To determine the function $C\left(\mathbf{y}_{k}\right)$, the partial derivative of Eq. (A.5) with respect to $y_{j^{\prime}, k}$ is

$$
\frac{\partial f}{\partial y_{j^{\prime}, k}}=(k-1) \frac{\rho_{k}\left(j^{\prime}\right) e_{k, s}}{\left(\sum_{j=2}^{\mathrm{r}_{\mathrm{m}}} \rho_{k}(j) y_{j, k}\right)^{k}}+\frac{\partial C\left(\mathbf{y}_{k}\right)}{\partial y_{j^{\prime}, k}} .
$$

From Eq. (A.4), we can write

$$
\frac{\partial C\left(\mathbf{y}_{k}\right)}{\partial y_{j^{\prime}, k}}=0\left(j^{\prime} \neq s\right), \frac{\partial C\left(\mathbf{y}_{k}\right)}{\partial y_{s, k}}=\epsilon \pi(k, s) .
$$

Therefore, we see that $C(\mathbf{y})=\epsilon \pi(k, s) y_{s, k}+C$, where $C$ is a constant. Since $f$ is a constant, let $C^{\prime}:=f-C$. From Eq. (A.5), we see that

$$
\begin{aligned}
& C^{\prime}=-\frac{e_{k, s}}{\left(\sum_{j=2}^{\mathrm{r}_{\mathrm{m}}} \rho_{k}(j) y_{j, k}\right)^{k-1}}+\epsilon \pi(k, s) y_{s, k} \\
& e_{k, s}\left(\mathbf{y}_{k}\right)=\left(\epsilon \pi(k, s) y_{s, k}-C^{\prime}\right)\left(\sum_{j=2}^{\mathrm{r}_{\mathrm{m}}} \rho_{k}(j) y_{j, k}\right)^{k-1} .
\end{aligned}
$$


From the initial condition $e_{k, s}(t=0)=\epsilon \pi(k, s)$, we see that $C^{\prime}=0$. Hence, we obtain

$$
e_{k, s}\left(\mathbf{y}_{k}\right)=\epsilon \pi(k, s) y_{s, k}\left(\sum_{j=2}^{\mathrm{r}_{\mathrm{m}}} \rho_{k}(j) y_{j, k}\right)^{k-1} .
$$

Using Eqs. (A. 3) and (A.6) for Eq. (A. 1), we obtain for $j \in\left\{2, \ldots, \mathrm{r}_{\mathrm{m}}\right\}, k \in\left\{2, \ldots, 1_{\mathrm{m}}\right\}$

$$
\frac{\mathrm{d} y_{j, k}}{y_{j, k}}=-\frac{h_{j}}{\sum_{i^{\prime}=2}^{1_{\mathrm{m}}} e_{i^{\prime}, j}} \mathrm{~d} t .
$$

We see that $y_{j, k^{\prime}}=y_{j, k}$ for $k^{\prime} \neq k$. Define $y_{j}:=y_{j, k}$ and $\mathbf{y}:=\mathbf{y}_{k}$. Hence, we obtain Eqs. (16) and (17) from Eqs. (A.3) and (A.6). Moreover, this differential equation becomes Eq. (13).

\section{A.2 Proof of Eqs. (14) and (15)}

Note that $\sum_{k=2}^{\mathrm{l}_{\mathrm{m}}} e_{k, s}=\sum_{\tilde{s}=1}^{s} r_{s, \tilde{s}}$. Using Eqs. (A. 1) and (A. 6) for Eq. (4), we obtain

$$
\begin{aligned}
\mathrm{d} r_{s, \tilde{s}}= & \tilde{s} \frac{r_{s, \tilde{s}+1}-r_{s, \tilde{s}}}{\sum_{i} e_{i, s}} \sum_{i=2}^{1_{\mathrm{m}}}(i-1) \frac{e_{i, s}}{l_{i}} \sum_{j^{\prime}=2}^{\mathrm{r}_{\mathrm{m}}} h_{j^{\prime}} p_{i, j^{\prime}} \mathrm{d} t \\
= & \frac{\tilde{s}\left(r_{s, \tilde{s}}(\mathbf{y})-r_{s, \tilde{s}+1}(\mathbf{y})\right)}{\sum_{i=2}^{l_{\mathrm{m}}} \lambda_{s}(i)\left(\sum_{j=2}^{\mathrm{r}_{\mathrm{m}}} \rho_{i}(j) y_{j}\right)^{i-1}} \sum_{i=2}^{1_{\mathrm{m}}}(i-1) \lambda_{s}(i) \\
& \cdot\left(\sum_{j=2}^{\mathrm{r}_{\mathrm{m}}} \rho_{i}(j) y_{j}\right)^{i-2} \sum_{j^{\prime}=2}^{\mathrm{r}_{\mathrm{m}}} \rho_{i}\left(j^{\prime}\right) \mathrm{d} y_{j^{\prime}}
\end{aligned}
$$

To solve Eq. (A.7), we define a substitution $H(\mathbf{y}):=$ $\sum_{i=2}^{\mathrm{l}_{\mathrm{m}}} \lambda_{s}(i)\left(\sum_{j=2}^{\mathrm{r}_{\mathrm{m}}} \rho_{i}(j) y_{j}\right)^{i-1}$, which gives

$$
\mathrm{d} H(\mathbf{y})=\sum_{i=2}^{1_{\mathrm{m}}}(i-1) \lambda_{s}(i)\left(\sum_{j=2}^{\mathrm{r}_{\mathrm{m}}} \rho_{i}(j) y_{j}\right)^{i-2} \sum_{j^{\prime}=2}^{\mathrm{r}_{\mathrm{m}}} \rho_{i}\left(j^{\prime}\right) \mathrm{d} y_{j^{\prime}} .
$$

Using this substitutions, Eq. (A-7) is written as

$$
\mathrm{d} r_{s, \tilde{s}}(H)=\tilde{s}\left(r_{s, \tilde{s}}(H)-r_{s, \tilde{s}+1}(H)\right) \frac{\mathrm{d} H}{H} .
$$

This equation is a first order linear differential equation. The solution is given by

$$
r_{s, \tilde{s}}(H)=H^{\tilde{s}}\left\{-\tilde{s} \int r_{s, \tilde{s}+1}(H) H^{-\tilde{s}} \frac{\mathrm{d} H}{H}+C_{s, \tilde{s}}\right\}
$$

for some constants $C_{s, \tilde{s}}$ to be determined from the initial conditions. These equations can be solved recursively, starting with $r_{s, s}$. We see that

$$
r_{s, \tilde{s}}(H)=\sum_{i \geq \tilde{s}}(-1)^{i+\tilde{s}}\left(\begin{array}{l}
i-1 \\
\tilde{s}-1
\end{array}\right) C_{s, i} H^{i} .
$$

This equation can be solved recursively, starting with $C_{s, s}$. Using initial conditions for Eq. (A.8), we see that

$$
C_{s, i}=\sum_{j \geq i}\left(\begin{array}{l}
j-1 \\
i-1
\end{array}\right) r_{s, j}(t=0) .
$$

Note that $H(t=0)=1$. Let $R_{s}$ be the number of check nodes of original degree $s$. We see that

$$
R_{s, j}(t=0)=j R_{s}\left(\begin{array}{c}
m \\
j
\end{array}\right) \epsilon^{j}(1-\epsilon)^{s-j} .
$$

Let the both sides be multiplied by $1 / E$. We obtain

$$
r_{s, j}(t=0)=\rho_{s}\left(\begin{array}{c}
s-1 \\
j-1
\end{array}\right) \epsilon^{j}(1-\epsilon)^{s-j}
$$

where $\rho_{s}=\sum_{i} \pi(i, s)$. From this equation and Eq. (A.9), we can write

$$
C_{s, i}=\rho_{s}\left(\begin{array}{c}
s-1 \\
i-1
\end{array}\right) \epsilon^{i} .
$$

(Use the identity $\left(\begin{array}{c}j-1 \\ i-1\end{array}\right)\left(\begin{array}{l}s-1 \\ j-1\end{array}\right)=\left(\begin{array}{c}s-1 \\ i-1\end{array}\right)\left(\begin{array}{c}s-i \\ j-i\end{array}\right)$ ). From this equation and Eq. (A. 8), we can write

$$
\begin{aligned}
r_{s, \tilde{s}}(\mathbf{y})= & \rho_{s}\left(\begin{array}{c}
s-1 \\
\tilde{s}-1
\end{array}\right)(\epsilon H(\mathbf{y}))^{\tilde{s}}(1-\epsilon H(\mathbf{y}))^{s-\tilde{s}} \\
= & \rho_{s}\left(\begin{array}{c}
s-1 \\
\tilde{s}-1
\end{array}\right)\left\{\epsilon \sum_{i=2}^{1_{\mathrm{m}}} \lambda_{s}(i)\left(\sum_{j=2}^{\mathrm{r}_{\mathrm{m}}} \rho_{i}(j) y_{j}\right)^{i-1}\right\}^{\tilde{s}} \\
& \cdot\left\{1-\epsilon \sum_{i=2}^{1_{\mathrm{m}}} \lambda_{s}(i)\left(\sum_{j=2}^{\mathrm{r}_{\mathrm{m}}} \rho_{i}(j) y_{j}\right)^{i-1}\right\}^{s-\tilde{s}} .
\end{aligned}
$$

Hence, we obtain Eq. (15).

Note that $\sum_{k=2}^{1_{\mathrm{m}}} e_{k, s}(\mathbf{y})=\sum_{\tilde{s}=1}^{s} r_{s, \tilde{s}}(\mathbf{y})$. Using Eqs. (15) and (17), we can write

$$
\begin{aligned}
r_{s, 1}(\mathbf{y})= & \sum_{k=2}^{l_{\mathrm{m}}} e_{k, s}(\mathbf{y})-\sum_{\tilde{s}=2}^{s} r_{s, \tilde{s}}(\mathbf{y}) \\
= & \epsilon \sum_{i=2}^{1_{\mathrm{m}}} \pi(i, s)\left(\sum_{j=2}^{\mathrm{r}_{\mathrm{m}}} \rho_{i}(j) y_{j}\right)^{i-1}\left[y_{s}-1\right. \\
& \left.+\left\{1-\epsilon \sum_{i=2}^{1_{\mathrm{m}}} \lambda_{s}(i)\left(\sum_{j=2}^{\mathrm{r}_{\mathrm{m}}} \rho_{i}(j) y_{j}\right)^{i-1}\right\}^{s-1}\right] .
\end{aligned}
$$

Hence, we obtain Eq. (14).

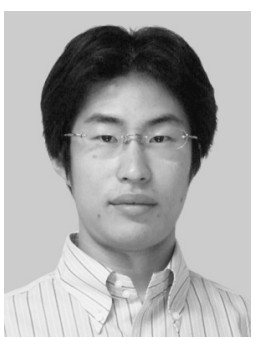

Takayuki Nozaki was born in Tokyo, Japan in 1986. He received B.E. degree in computer science from Tokyo Institute of Technology, Tokyo, Japan, in 2008. He is currently pursuing the M.E. degree in Department of Communications and Integrated Systems at Tokyo Institute of Technology. His research interests are codes on graphs and iterative decoding algorithms. 


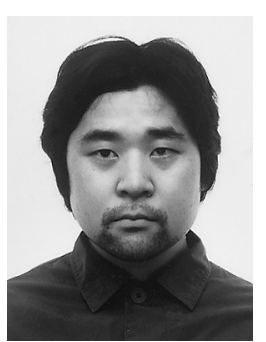

Kenta Kasai received B.E., M.E. and Ph.D. degrees from Tokyo Institute of Technology in 2001, 2003 and 2006, respectively. Since April 2006, he has been an research associate in the Department of Communications and Integrated Systems, Graduate School of Science and Engineering, Tokyo Institute of Technology. His current research interests are codes on graphs and iterative decoding algorithms.

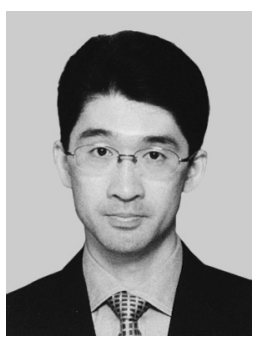

Tomoharu Shibuya received B.E., M.E., and $\mathrm{Ph} . \mathrm{D}$. degrees in electrical and electronic engineering from Tokyo Institute of Technology, Tokyo, Japan, in 1992, 1994 and 1999, respectively. He joined Tokyo Institute of Technology in 1994 as a research associate in the Department of Electrical and Electronic Engineering, and served as a research associate in the Department of Communications and Integrated Systems from 1999 to 2003. From 2003, he served as an associate professor in R\&D Department, National Institute of Multimedia Education. Since 2008, he has been an associate professor in the Department of Mathematics in Sophia University. He received the Young Engineer Award from the IEICE of Japan in 1997 and the Ericsson Young Scientist Award in 2005. His research interests are error control coding, information theory and communication theory. He is a member of IEEE, SITA, IPSJ, and MSJ.

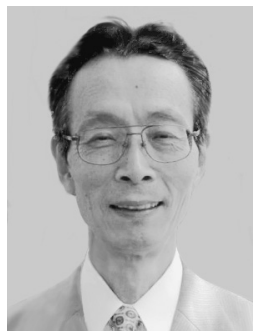

Kohichi Sakaniwa received B.E., M.E., and $\mathrm{Ph} . \mathrm{D}$. degrees all in electronic engineering from the Tokyo Institute of Technology, Tokyo Japan, in 1972, 1974 and 1977, respectively. He joined the Tokyo Institute of Technology in 1977 as a research associate and served as an associate professor from 1983 to 1991. Since 1991 he has been a professor in the Department of Electrical and Electronic Engineering, and since 2000 in the Department of Communication and Integrated Systems, Graduate School of Science and Engineering, both in the Tokyo Inst. of Tech. From November 1987 to July 1988, he stayed at the University of Southwestern Louisiana as a Visiting Professor. He received the Excellent Paper Award from the IEICE of Japan in 1982, 1990, 1992 and 1994. His research area includes Communication Theory, Error Correcting Coding, (Adaptive) Digital Signal Processing and so on. Dr. Sakaniwa is a member of IEEE, Information Processing Society of Japan, Institute of Image Information and Television Engineers of Japan, and Society of Information Theory and its Applications. 